in conjunction with Mr. Dillwyn. But Mr. Mottley's untimely death in the Malay insurrection of 1860 put a stop to the publication, though his Banjermassing collection was subsequently catalogued by Mr. Sclater in the Zoological Society's " Proceedings."

From these and various other authorities, of which a complete account is given in the introduction to the work, and from the study of Doria's numerous series, Dr. Salvadori has compiled his list of 392 species of Bornean birds. Their synonymy is very fully stated, and the localities are completely given, whilst descriptions and remarks of various characters are added when necessary. Of the 392 species of Bornean birds, filty-eight, Dr. Salvadori tells us, are peculiar to the island, whilst the remainder are found also in Malacca and Sumatra, or have a still wider distribution. With these last-named countries it is, as already pointed out by Lord Walden,* that Borneo has a most intimate relation, upwards of 250 species being common to these three localities. These and many other facts relating to the ornithology of Borneo are well put together by our author in this excellent memoir, on which it is obvious great labour has been bestowed. The volume is rendered still more complete by an outline map of Borneo and the adjacent islands, and by several coloured plates of the rarer species of birds, amongst which the extraordinary shrike-like form called Pityriasis gymnocephala forms a conspicuous object. Dr. Salvadori's work is thus an indispensable addition to a naturalist's library.

\section{PHENOLOGICAL PHENOMENA†}

T NDER the title given below a pamphlet has just been issued containing instructions for the correct observation of the first appearance of insects, birds, and plants in flower in any locality. We recommend it to the attention of all who have opportunities of making such observations, and there are thousands who have. If a host of observers could be enlisted in this work, and if they adhered faithfully to the instructions given in the pamphlet, they would not only find a new source of real pleasture and instruction, but would certainly make large contributions to our knowledge of natural history.

$A$ list is given of ninety-seven plants, insects, and birds to be observed, with a set of general rules, approximate phenological dates, and special remarks and suggestions in connection with the various divisions. Those in botany are drawn up by the Rev. T. A. Preston, F.M.S. ; in entomology, by Mr. R. McLachlan, F.L.S ; and in ornithology, by Prof. A. Newton, F.R.S. Each of them presents a series of notes on various individual plants and animals, and Prof. Newton has some general remarks in his own department, from which we make the following extracts :-

"It constantly happens, especially among the earlier birds of passage in spring, that they will for some days haunt one particular spot before appearing in others or generally throughout the district. I myself knew a particular reach of a river which was yearly frequented by the Sand-Martin for nearly a week or ten days before examples of that species were to be seen elsewhere in the vicinity. I also knew a parish in which the Chiffchaff always bred, but not for a month or six weeks after it had arrived in many of the neighbouring parishes was its note to be heard within the limits of that particular parish. I could easily cite other cases of like nature, but many if not most observers of birds from their own experience will bear me out in this. It follows, therefore, that to render the proposed observations trustworthy, an

Ibis, 1872, p. $36 \mathrm{r}$.

Instructions for the Observation of Phenological Phenomena, prepared at the request of the Council of the Meteorological Society by a Conference consisting of Delegates from the following Societies, viz: Koyal Agricultural Society, Royal Botanic Society, Royal Dublin Society, Royal Horticultura Society. observer of any fact connected with birds should set down the exact locality at which it occurred, even if it be but a few miles' distance from his own station, and if possible again record the fact when it recurs there; or vice versá. Otherwise there will naturally be a risk of considerable error, but an attentive observer will probably soon come to find out the localities in his neighbourhood which are first visited by any particular kind of bird, and after a few years' experience the double observation will very likely prove unnecessary."

After giving some notes on a number of individual birds, Prof. Newton goes on :-

"Nearly all the observations above suggested can be made or collected by most residents in the country generally, and even by some who live in towns; but such observers as dwell at or near the seaside--and especially not far from the stations chosen by various sea-fowls for their breeding quarters-are recommended to keep watch for their arrival and departure. It has been frequently asserted that many of these birds, as the Guillemot, Puffin, Razorbill, and certain Gulls, resort to and quit their stations punctually on a particular day, regardless of the state of the weather; and if such statements are correct, the facts which render the birds independent of meteorological conditions seem to deserve attention. In some cases the assistance of lighthouse-keepers, if sought, would probably conduce to the success of the inquiry, as they almost always take an interest in the doings of their feathered neighbours. Lighthouse-keepers, it is believed, could also furnish valuable information as to the extraordinary flocks of migrant birds which occur by night at uncertain intervals. These flocks consist of a very heterogeneous assemblage, and it is seldom that the particular kinds can be identified except by the victims that may be found next morning lying dead beneath the glasses against which they have dashed themselves. Similar flocks are occasionally observed inland, and chiefly over or near large towns, whither it may be supposed they have been attracted by the glare of the street lamps. In these latter cases it is seldom that examples are procured to show of what species the flock was composed, but the mere fact of its occurrence is always worthy of record with the precise hour at which the birds were heard, in weather report. The cries, whistling, and screams of the birds, sometimes even the sound of their wings, are often enough to attract the attention of the most unobservant; and, as far as I know, these miscellaneous flocks only occur on perfectly still pitch-dark nights, with a comparatively high temperature and a falling barometer-circumstances that point to an atmospheric cause of the wonderful concourse.

"A connection between the habits of birds and meteorological conditions is popularly believed to exist in the case of the Green Wocdpecker, the frequent cry of which is said to presage sain; but I have failed to find that this is so. The Redbreast, on the other hand, when singing from an elevated perch at evening, is said to be an unfailing prophet of a fine day on the morrow, while if its parting song be uttered from a lower station bad weather is supposed to follow. As far as my own experience goes, the only connection between changes of weather and the habits of birds (omitting of course hard frost and deep snow, the effects of which are cbvious) is, that many birds seem to be more alert, or 'wilder,' as the sportsmen say, for a day or two before a heavy downfall; I have observed this with partridges, plovers, and snipes."

We recommend all our readers to procure these "In. structions."

\section{INSTITUTION OF NAYAL ARCHITECTS}

$A^{T}$ the annual meeting of this Institution last? week, three papers of interest to the scientific world were read and discussed. All three of these papers bore upon 
the subject of waves, which is at present occupying so much the attention of all those who, both in this country and abroad, are endeavouring, by researches into their forms and habits, to improve the theory of Naval Architecture.

The first paper was on a proposed method of obtaining the outlines of deep-sea waves, by Mr. W. W. Rundell, the secretary of the Liverpool Underwriters' Association. The important part which photography has recently played in the observations on the Transit of Venus, and the assistance which it has thus rendered to astronomy, led Mr. Rundell to consider whether it might not also be employed to determine the forms of waves and so supply data for obtaining their chief components. The application which Mr. Rundell proposes consists of a system of poles about 36 feet in length, painted with alternate bands of red and blue, each band being a foot wide. These poles are spaced I 5 feet apart and loosely coupled at one end to yards or spare spars extending to a length of about 600 feet. A similar system of poles intersects the first system at intervals of 90 feet, the different parts being connected together, in moderate weather, while floating on the surface of the water. Weights being attached to the spars would cause the poles to sink until only about 12 feet of their length was visible above the water. Mr. Rundell proposes, by the aid of photography, to take pictures of the outlines of waves seen against this system, the photographs being taken either from the crosstrees of a man-of-war or from some elevated position such as the Fastnet, or Skellig Lighthouses. Mr. Rundell thinks that thus the complete history of a gale might be photographically recorded. Mr. Froude, however, seemed to think that there would be greater difficulties to encounter than $\mathrm{Mr}$. Rundell imagined.

The next paper, by Mr. Froude, was a description of the graphic integration on the equation of a ship's rolling, including the effect of resistance. Mr. Froude first pointed out that the commonly employed methods of graphic integration, i.e. the semi-geometrical processes by which the solution of intraciable mathematical problems is effected, do not readily lend themselves to the treatment of a problem in which the forces which govern the move. ments of the body arise afresh at each instant, as the direct and indirect effects of the very movements they are creating, but that his method is perfectly capable of dealing with this circumstance.

The two principal forces taken account of in this method are the ship's "righting force " or "moment" as dependent on her inclination relatively to the wave slope at each instant, taking into consideration any speciality in her curve of stability; and the resistance she experiences while in motion, as dependent on her angular velocity. Taking the equation of rolling motion to be integrated is, in its most complete form, as follows :-

$$
-\frac{d^{2} \theta}{d t^{2}}=\frac{\pi^{2}}{\mathrm{~T}^{2}}\{f(\theta-\theta)+\mathrm{R}\}
$$

Here $\theta$ is the ship's absolute inclination, $\theta^{\prime}$ the inclina. tion of the wave, and $\therefore\left(\theta-\theta^{\prime}\right)$ is her inclination relatively to the wave slope, or the ship's "relative inclination;" the term $f\left(\theta-\theta^{\prime}\right)$ signifies that function of the relative inclination which in the curve of stability is assigned to the particular inclination, and expresses the righting moment of the ship when so inclined.

$T$ is the time, in seconds, occupied by the ship in performing a single swing when rolling to moderate angles in still water, being half of what is commonly called the "metacentric period."

$\mathrm{R}$ is the effective "moment of resistance" which the ship is at the instant experiencing when rolling with her existing angular velocity, its elementary signification being homogeneous with that in the ship's curve of stability, in which $f\left(\theta-\theta^{\prime}\right)$ stands for the righting moment. In both terms, alike, these elements consist in effect of "so many foot-tons $\times \frac{g}{\mathrm{~W} \rho} \rho^{2}$ " where $\mathrm{W}$ is the ship's weight in tons, $\rho$ her radius of gyration expressed in feet, as $g$ also usually is. The abstract value of $R$ is

$$
k_{1} \frac{d \theta}{d t} \pm k_{2} \frac{d \theta^{2}}{d t^{2}}
$$

where $k_{1}$ and $k_{2}$ will have values appropriate to the particular ship in question; and observe that the \pm sign must be understood to mean that the sign of the second term, which, being a square, would in itself be always positive, must change signs in company with the first term.

A base line being taken to represent time, and divided into equal spaces representing small unit-intervals of time, $\Delta_{1} t, \Delta_{2} t$, \&c., the inclination at each instant, whether of the ship or of the wave, are to be expressed as ordinates to a scale of degrees; those above the base line being positive, and those below it negative. A "curve of wave slopes" being drawn, the ship's absolute inclinations, which grow out of the circumstances, as time (and the varying wave slopes which time brings) proceeds, by Mr. Froude's method of graphic integration, are represented by a curve analogous to the "curve of wave slopes" in general character. This curve which gradually grows out of the integration Mr. Froude calls the "curve of rolling" or the "curve of inclinations." The difference between the ordinates of these two curves, at any instant, gives the ship's relative inclination at that instant on which the righting force depends. The angular velocity of the ship's change of inclination is obviously expressed by the tangential direction of the curve, and this circumstance is of essential importance in the process by which the curve is deduced.

To carry out the process two auxiliary curves have to be introduced :-

I. The "ship's curve of stability," which supplies, as has been explained, her righting moment, as due to her relative inclination at any instant. In this, the base is formed of a scale of angles, this scale being the same as in the "curve of wave slopes" and the "curve of inclinations." The ordinates corresponding with given inclinations express the righting moments at those inclinations to the scale which is employed in the graphic process.

2. The "curve of resistance," which supplies the moment of resistance experienced by the ship when moving with any given angular velocity.

As has been already stated, the conditions are-

$$
\mathrm{R}=k_{1} \frac{d \theta}{d t}+k_{2} \frac{d \theta^{2}}{d t}
$$

The first of these terms is expressed by a straight line, and the second by a parabola which takes that straight line as its base.

Turning to the employment of these data in the geometrical solution of the dynamical equation, by grouping the force terms under the single symbol $\phi$, we may write the equation thus :-

$$
d(d \theta): d t=\phi: \frac{\mathrm{T}^{2}}{\pi^{2} d t}
$$

Substituting for the differential terms, small quantities virtually infinitesimal-

$$
\Delta(\Delta \theta): \Delta t=\phi: \frac{T^{2}}{\pi^{2} \Delta t}
$$

where $\Delta t$ is the unit of space taken in the curve of wave slopes.

By a simple geometrical contrivance this ratio is utilised by drawing a base line each way from the foot of the vertical axis in the "curve of resistance," which measured by the time scale $=\frac{\mathrm{T}^{2}}{\pi^{2} d}$, ending at $+\mathrm{P}$ and
$-\mathrm{P}$. Through the end of this line the inclination $\theta_{0}$ is set off with a parallel ruler from the "curve of wave slopes." The height $p$ at which this line cuts the directrix is proportional to the angular velocity. 
Now, $\Delta(\Delta \theta)$, the difference of inclination which we wish to find, as has been shown,

$$
=\Delta t \cdot \phi \frac{\pi^{2} \Delta t}{T^{2}}
$$

and $\phi$ consists of $f\left(\theta-\theta^{\prime}\right)+\mathrm{R}$, of which the former is the ship's righting moment, and the second her moment of resistance.

Thus we can find $R$ from the angular velocity, $\theta$ from the "curve of wave slopes," and $\theta^{\prime}$ absolutely at the beginning of the first interval and approximately at the end of the subsequent time intervals.

The difference between the exact ordinate length of the two curves at $t_{0}$ and approximately estimated length at $t_{1}$ is applied by dividers to the line of abscissæ, and hence is obtained the value of $\theta-\theta^{\prime}$ and therefore the corresponding ordinate gives $f\left(\theta-\theta^{\prime}\right)$.

The sum of $\left(\theta-\theta^{\prime}\right)$ and of $\mathrm{R}$ is taken as an ordinate of the "force curve" at each point, and by connecting the tops of these ordinates we have a close approximation to the first segment of the force curve. The ordinate length of $\phi$ being now obtained, some necessary correction being made, if the line $P_{1} p$ be now drawn, the difference of its tangential inclination from that of $P_{0} p$ represents with close approximation (indeed, exactly, if the provisional estimate has been judiciously made) the change of velocity which has ensued during $\Delta_{1} t$, and a line parallel to $P_{0} p$ will be the tangent to the curve of inclination at $t_{1}$. Draw, therefrom, $b b$ as the tangent across the ordinate $t_{1}$ at such a height that it shall intersect the previous tangent at the middle part of $\Delta_{1} t$. This height is the first approximate value of the ordinate to the curve of inclinations at that point.

By carrying out this method the whole curve of inclinations is obtained.

This description of Mr. Froude's paper is necessarily very imperfect, through our being obliged to leave out the small corrections, which without figures would be unintelligible. But it is sufficient to show his extremely neat and simple way of drawing a curve which shall determine a ship's absolute inclinations.

The third paper was by Mr. B. Tower, on a method of obtaining motive power from wave motion. He said that this inquiry originated with Mr. Deverell, who came home from the antipodes for the purpose of promulgating it. Mr. Deverell's proposition was to suspend a heavy weight on board a ship by means of springs, and to obtain motive power by the oscillation of this weight through a distance not more than the height of the waves. It however appeared to Mr. Tower that since the centrifugal force of wave motion in a vertical direction is alternately

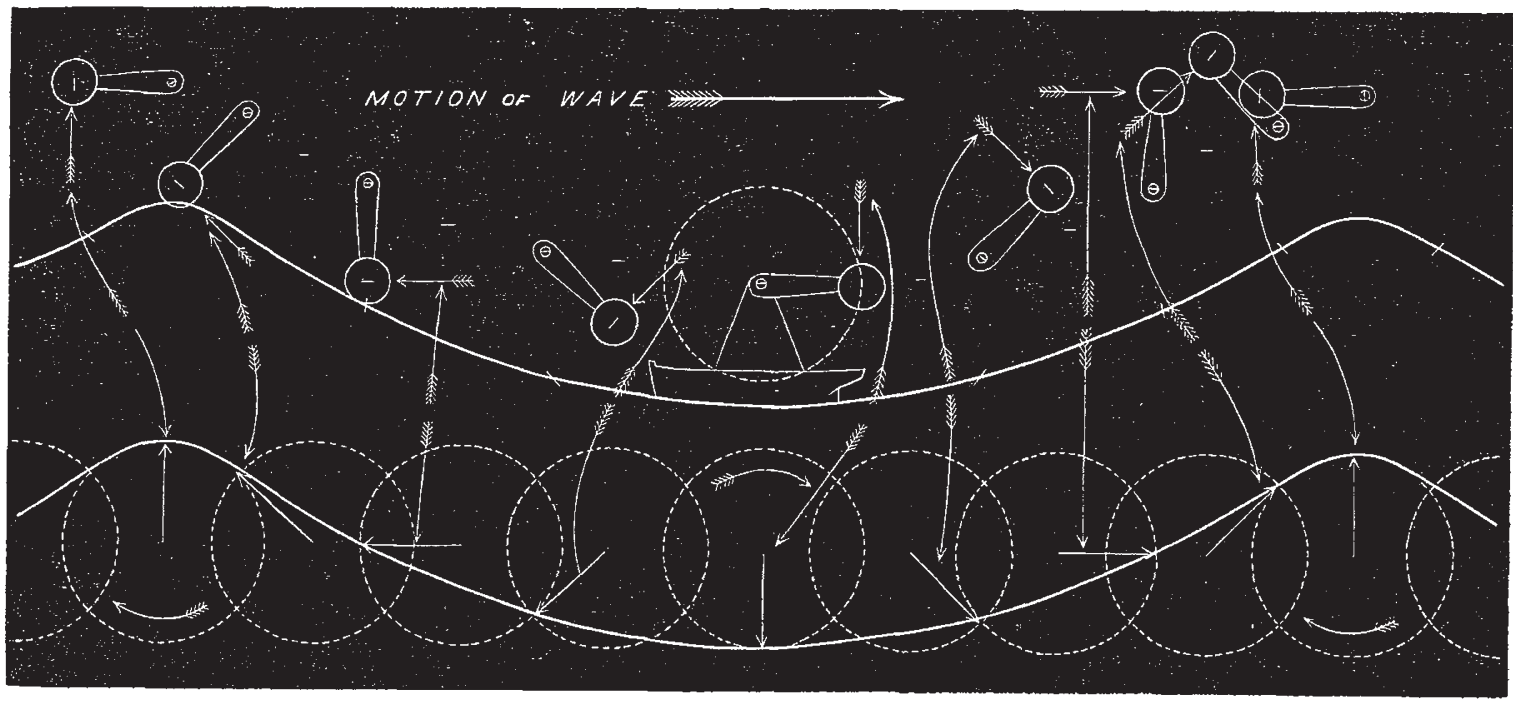

added to and subtracted from, the force of gravity thereby causing a virtual variation of the intensity of that force, the question might be broadly stated as follows :-

Supposing the force of gravity to vary in intensity at regular intervals, that is, to become alternately greater and less than its normal amount, what is the best means to obtain the maximum amount of energy from a given weight oscillating under the influence of these variations? For example, supposing the force of gravity to be for three seconds one-fifth greater, and for the next three seconds one-fifth less than its natural intensity, and suppose that we have a weight of five tons suspended by a spring, with an infinitely open scale, so that the spring will continue to exert a uniform upward force of five tons, no matter how far the weight moves up and down, it is clear that during the three seconds' interval, during which gravity is one-fifth more than its normal intensity, the five-ton weight will virtually weigh six tons, and will thus exceed the upward force of the spring by a downward force of one ton; in the same way, when the force of gravity is one-fifth less, the weight will only weigh four tons, and the spring will then exert an unbalanced upward force of one ton. Now, as energy or power is defined as force moving through distance, it is clear that the quantity of energy or power to be obtained by this system will depend on the distance through which this weight is caused to move during each successive variation of gravity. Thus, supposing that during the plus interval it moves downwards through one foot, and during the minus interval it moves upwards through one foot, it is clear that during each of these intervals it will exert a force of one ton moved through one foot-that is, one foot-ton; but if, instead of one foot, it moves through ten feet, it will exert ten times the power-that is, ten foot-tons ; or if it moved through roo feet, it would exert roo foot-tons during each interval of three seconds.

The first experiments Mr. Tower made with a model apparatus constructed on these principles showed him that the best arrangement would be to put a weight on the end of a revolving arm, whereby the centrifugal force of the wave motion might be utilised as well as the rising and falling motion.

The diagram shows the position of the vessel and of its revolving arm at all parts of a wave; the arrows show 
the direction of the centrifugal force of the wave motion according to the generally received theory. This force is upwards at the crests, downwards in the hollows, and horizontal midway between the crests and hollows. If the weighted arm is compelled to assume successive angular positions, so that it is always at right angles to the force, it is evident that the force will be continually acting to cause the arm to rotate. It is easy to see how the work is taken out of the waves, for when the vessel is descending, the weight is performing the upper half of its revolution, and is consequently exerting an upward centrifugal force; and when the vessel is ascending, the centrifugal force is pushing down and resisting the vessel's ascent, so that the revolving weight affords a resistance against which the vessel can push just as if it were a fixed point in space. The shaft of the revolving weight can be made to turn a screw in the stern of the vessel by means of a proper system of gearing, and by a delicate arrangement of electric brakes and hydraulic accumulators, Mr. Tower proposes to regulate the revolving arm so as always to keep it at right angles to the centrifugal force of the waves.

\section{THE FARADAY LECTURE}

I AST Thursday, as our readers know, Dr. A. W Hofmann, of Berlin, delivered at the Royal Insti tution the Faraday Lecture of the Chemical Society, his subject being "Liebig's Contributions to Experimental Chemistry." The audience included the Prince of Wales Dr. Odling occupied the chair. The dinner on Friday at Wiliis's Rooms was probably one of the most remarkable scientific dinners that have taken place for some years, there being about 180 present, nearly all of them wellknown men of science. Dr. Hofmann made a noble appeal on behalf of the recognition of the high value of pure scientific research; and Prof. Huxley acknowledged ha: while in scientific ideas we might be abreast of the Germans, yet the latter undoubtedly excelled us in the amount of their scientific work.

Dr. Hofmann in his lecture began by pointing out that Faraday belonged by the universality of his genius to all civilised countries, and the council of the Chemical Society had ordained that all countries should be asked to join in rendering homage to the greatest experimental thinker among mankind. On the present occasion Germany had been invited to take part in this international tribute, and it was a great honour for him to interpret his country's homage. His illustrious teacher and lamented friend Justus ron Liebig was a master mind like Faraday, and Liebig's is the name and figure alone fitted to stand on equal terms beside Faraday. But to speak of Liebig only we must proclaim him one of the greatest contributors to chemistry at large, while of organic chemistry he was the founder. It is not only by the discovery of new facts that he was distinguished, but by the conception of general laws which illustrate both organic and inorganic chemistry. By the great types of composition which under the name of "radicles" he first spoke of, and by the researches to which these led, he guided not only his contemporaries, but will guide succeeding ages. He was also the first to found in Europe the great system of practical education. It was at Giessen he organised the first great laboratory of experimental instruction; and if we now admire magnificent temples of science, let us not forget that we owe them to him as their originator. He called around him distinguished students, many of whom were raised now to exalted positions by their talents and learning, and many of whom the lecturer saw before him; they would not be wanting in the tribute of heartfelt reverence to their great master. By his keen insight into chemical analogy he marked out the way of chemical research, and he also showed how to keep up the supply of human hearts and minds to prosecute his work. He provided arms, and soldiers to wield them.

There is no greater proof of the fecundity of genius than that it enriches the storehouse of science with its discoveries, and at the same time provides the means of ulterior conquest in ages yet to come. Which of us returning tomorrow to his lonely post in the laboratory could not feel cheered by the example of such men as Liebig and Faraday? It was the habit of Liebig to trace laws in their furthest results, and their applicability to promoting the practical welfare of mankind. No one has in this way more enriched us. Liebig's labours in abstract science bore fruit in the useful arts. $\mathrm{He}$ materially elucidated great industries, the manufacture of fulminating compounds and prussiate of potash, for instance, together with materials of the most important use in the manufacture of the precious metals, and silver-coated mirrors, so preferable for purposes of science and adornment to the old mercury-backed glasses. Illustrans commoda vitae, he never let slip any occasion of promoting the good of his fellow-creatures. His penetrating philosophy could not remain a stranger to the profound secrets of life based upon chemical change. He revealed the dependence of plants upon the chemical composition of the soil and air. $\mathrm{He}$ studied also the laws of nutrition and development of the animal body. In the former of these branches he was crowned with the greatest success. He began in 1840 by his work on agriculture and zoology, written in compliance with the request of the British Association at their 1837 meeting at Liverpool, and he followed it up by his work on husbandry. His labours resulted in the establishment of the philosophy of agricultur, and ranked him with Lavoisier, who showed in the last century how the vegetable stands between the mineral and the animal, and collects from the former world food for the latter. Lavoisier was followed by Humphry Davy, and after him came Liebig. They are the three great lawgivers of modern agriculture.

It was in 1842 that Liebig, passing onward from food producers to animals, brought out his work on the subject, and it may be taken as a result of his work that the superabundant animal food of thinly-peopled parts of the earth has been, by a fast developing industry, brought over to guard Europe against the pinch of want. Those who are engaged in the curative art must bear him gratitude for the discovery of chloroform; nor will they forget chloral, the benign influences of which will even induce sleep, and rank it among the most sublime agencies placed at the disposal of therapeutics by chemical art.

He had, the lecturer said, selected but a few' illustrations, which would give them a better idea than any long explanations by him of Liebig's voluminous life-work, and he asked them to accompany him in a rapid view of Liebig's memorable contributions to chemistry proper. But Liebig's contributions to the Royal Society's library were, in 1863,317 in number, and 283 were entirely by himself. When the lecturer's pupils in Berlin heard that he had been entrusted with this lecture, they produced all the substances which Liebig at any period of his career more particularly illustrated; these preparations now crowded the table.

The first achievement he would allude to was that which, whether or not his most brilliant discovery, contributed most to facilitate the labours of chemists, and was the main source of the marvellous development of organic chemistry-analysis by combustion of organic bodies and the determination of their carbon by his form of measurement. Not one there but had determined the molecular weight of bodies, but they might not be aware that we are indebted for the process to Liebig. He never published any particular paper on the subject, but merely communicated his method in a paper on another chemist's researches. Its merit is simplicity, like that of his air analysis, That an alkaline solution of pyrogenic acid 